mit einer Abnahme der Fluoreszenzdauer der kurzwelligen Komponente verbunden ist. Will man die Beobachtungen am Acridinorange in diesem Sinne deuten, so kommt man zu dem Schluß, daß in der Lösung nur zwei Partner vorhanden sind, wahrscheinlich Monoionen (mit grüner Fluoreszenz) und Diionen (mit roter Fluoreszenz). Gegen diese Annahme spricht aber der spektroskopische Befund.
Die Untersuchungen wurden im Rahmen eines Forschungsauftrages der Deutschen Forschungsgemeinschaft durchgeführt, der ich an dieser Stelle für die Bereitstellung von Mitteln herzlich danke.

Besonders danke ich auch Herrn Prof. Dr. W. Hanle für sein förderndes Interesse, sowie Herrn Prof. Dr. G. Scheibe und Herrn Dr. Zanker für wertvolle Diskussionsbemerkungen. Der Freundlichkeit des Letztgenannten verdanke ich auch einen Teil des verwandten reinen Acridinorange.

\title{
Über den Temperaturanstieg in thermischen Reaktoren bei Ausfall der Kühlung
}

\author{
Von R. Schulten und H. Gaus \\ Aus dem Max-Planck-Institut für Physik, Göttingen \\ (Z. Naturforschg. 9a, 1039-1043 [1954]; eingegangen am 1. Oktober 1954)
}

Es wird eine Formel für den maximal möglichen Temperaturanstieg im Inneren eines Kernreaktors bei Ausfall der Kühlung und der Kontrollmechanismen abgeleitet.

$\mathrm{K}$ ernreaktoren können aus Materialgründen nur mit einer bestimmten Maximaltemperatur betrieben werden. Beim stationären Betrieb eines Reaktors ist durch die Stellung der Kontrollstäbe eine bestimmte Arbeitstemperatur festgelegt, die unabhängig von der entnommenen Leistung ist. Sie muß jedoch aus Gründen der Sicherheit unterhalb der Maximaltemperatur liegen. Die Leistung wird bei Reaktoren durch ein Kühlsystem entnommen, das die bei den Kernspaltungen frei werdende Wärme mittels eines durchlaufenden Kühlmittels heraustransportiert. Ein plötzliches Versagen der Kühlanlage würde den Reaktor zu einem praktisch wärmeisolierten System machen, so daß die zunächst noch erzeugte Energie einen Temperaturanstieg des Reaktors bewirkt. Dieser Temperaturanstieg (und eventuell die Wirkung eines Kontrollmechanismus) bewirkt dann ein Abreißen der Kettenreaktion. Die noch vorhandenen Neutronen werden vom Reaktor absorbiert, was eine weitere Temperaturerhöhung bewirkt. Besonders starke Temperaturerhöhungen können dann eintreten, wenn gleichzeitig mit der Kühlung die Kontrollapparatur versagt.

Für die Berechnung des Temperaturanstieges ist die Frage von Bedeutung, welchen Einfluß das Ausfallen der Kühlung auf die Reaktivität hat. Das durch den Reaktor fließende Kühlmittel kann einerseits durch Neutronenabsorption die Kettenreaktion hemmen, andererseits sie auch fördern, indem es die Neutronen abbremst und so die Moderatorwirkung verstärkt. Wirkt das Kühlmittel als Neutronenabsorber hemmend auf die Kettenreaktion ein, so wird die Reaktivität bei einer eventuellen Konzentrationsminderung des Kühlmittels ansteigen, der Reaktor ist inhärent instabil. Wenn umgekehrt das Kühlmittel durch eine Vergrößerung der Moderationskraft den Kettenproze $\beta$ begünstigt, so wird bei einer Konzentrationsverminderung des Kühlmittels die Reaktivität abnehmen; der Reaktor ist inhärent stabil. Dieser Effekt ist vor allem bei solchen Kühlmitteln zu berücksichtigen, die vornehmlich aus leichten Kernen bestehen. In dieser Arbeit soll nun folgende Frage beantwortet werden:

Wie groß ist die Zunahme der Innentemperatur eines Reaktors, der bis zum Zeitpunkt $t=0$ mit einer bestimmten Leistung $Q_{0}$ und einer Temperatur $T_{0}$ stationär betrieben wurde, wenn die Kühlung vollständig ausfällt und damit ein gleichzeitiger Anstieg (oder Abfall) der Reaktivität um $\left(\delta k_{1}-\delta k_{0}\right)$ verbunden ist?

Ein geringer Teil der Reaktorleistung (ca. 5\% wird in Form einer verzögerten $\gamma$ - bzw. $\beta$-Strahlung der Spaltprodukte geliefert. Der Einfluß dieser Strahlung auf den Temperaturanstieg, der nur bei sehr großem Neutronenfluß von Bedeutung ist, wird zunächst nicht berücksichtigt und im letzten Abschnitt diskutiert. 


\section{Die Gleichungen der Reaktorkinetik}

Das zeitliche Verhalten des Reaktors wird beschrieben durch die Gleichungen ${ }^{1}$ :

$$
\begin{aligned}
\frac{\mathrm{d} n}{\mathrm{~d} t} & =\frac{1}{t_{0}} k_{\mathrm{eff}}(1-\beta) n-\frac{1}{t_{0}} n+p P_{\mathrm{s}} \sum_{i} \lambda_{i} C_{i}, \\
\frac{\mathrm{d} C_{i}}{\mathrm{~d} t} & =\beta_{i} \frac{k}{p} \frac{P_{\mathrm{t}} n}{t_{0}}-\lambda_{i} C_{i} .
\end{aligned}
$$

$n=$ thermische Neutronendichte $=$ Anzahl der Neutronen pro $\mathrm{cm}^{3}$;

$t_{0}=$ mittlere Lebensdauer der Neutronen im Reaktor;

$k_{\text {eff }}=$ Vermehrungsrate für den endlichen Reaktor;

$\beta=$ Bruchteil der verspäteten Neutronen;

$p=$ Resonanzentkommwahrscheinlichkeit;

$P_{\mathrm{t}}=$ Wahrscheinlichkeit, daß ein thermisches Neutron im Reaktor verbleibt;

$P_{\mathrm{s}}=$ Wahrscheinlichkeit, daß ein schnelles Neutron im Reaktor verbleibt;

$\lambda_{i}=$ reziproke mittlere Lebensdauer der verspäteten (latenten) Neutronen;

$C_{i}=$ Konzentration der verspäteten (latenten) Neutronen der $i$-ten Art;

$\beta_{i}=$ Bruchteil der verspäteten Neutronen der Sorte $i$;

$k=$ Vermehrungsfaktor des unendlichen Reaktors.

Gl. (1 a) stellt die Änderung der Dichte der thermischen Neutronen, gemittelt über den gesamten Reaktor, dar. Der zweite Summand beschreibt die Anzahl der pro $\mathrm{cm}^{3}$ und sec eingefangenen Neutronen. Der erste Summand gibt die dabei entstehenden Neutronen an, abzüglich des Anteils $\beta$ der verspäteten (latenten) Neutronen. Der dritte Summand endlich gibt die pro sec und $\mathrm{cm}^{3}$ entstehenden verspäteten Neutronen wieder.

Die Gl. $(1 \mathrm{~b})$ beschreibt die zeitliche Änderung der verspäteten Neutronen der $i$-ten Art. $P_{\mathrm{t}} n / t_{0}$ ist die Anzahl der eingefangenen thermischen Neutronen, von denen der Bruchteil $\beta_{i} k / p$ zu der Entstehung der latenten Neutronen pro sec führt. Der zweite Summand gibt ihre Zerfallsrate pro $\mathrm{cm}^{3}$ und sec an.

Es wird vorausgesetzt, daß der Reaktor bis zum Zeitpunkt $t=0$ stationär betrieben wird. Dann ist bis $t=0$

$$
\mathrm{d} C_{i} / \mathrm{d} t=0 .
$$

Es folgt daraus die konstante Konzentration der latenten Neutronen:

$$
C_{i 0}=\beta_{i} P_{\mathrm{t}} k n_{0} / p \lambda_{i} t_{0} .
$$

${ }^{1}$ S. Glastone u. M. C. Edlund, Nuclear Reactor Theory, Macmillan \& Co. Ltd., London 1953. $n_{0}=$ stationäre Neutronendichte vor dem Zeitpunkt $t=0$,

$C_{i 0}=$ stationäre Dichte der latenten Neutronen der Sorte $i$.

Vom Zeitpunkt $t=0$ an ist

$$
\begin{aligned}
C_{i}(t) & =\beta_{i} \frac{P_{\mathrm{t}} k}{P t_{0}} \exp \left(-\lambda_{i} t\right) \int_{0}^{t} \exp \left(\lambda_{i} t^{\prime}\right) n\left(t^{\prime}\right) \mathrm{d} t^{\prime} \\
& +C_{i 0} \exp \left(-\lambda_{i} t\right)
\end{aligned}
$$

eine Lösung der Gl. $(1 \mathrm{~b})$. Durch Einsetzen von $C_{i 0}$ aus Gl. (2) in Gl. (3) und eine einmalige partielle Integration erhält man

$$
\begin{aligned}
C_{i}(t) & =\frac{\beta_{i}}{p} \frac{P_{\mathrm{t}} k}{t_{0} \lambda_{i}} \\
& \cdot\left\{n(t)-\int_{0}^{t} \exp \left[-\lambda_{i}\left(t-t^{\prime}\right)\right] \frac{\mathrm{d} n\left(t^{\prime}\right)}{\mathrm{d} t^{\prime}} \mathrm{d} t^{\prime}\right\} .
\end{aligned}
$$

Setzt man diesen Ausdruck in Gl. (1 a) ein, so erhält man für die Änderung der Neutronendichte nach dem Zeitpunkt $t=0$

$\frac{\mathrm{d} n}{\mathrm{~d} t}=\frac{1}{t_{0}}\left\{\left(k_{\mathrm{eff}}-1\right) n(t)-k_{\mathrm{eff}} \int_{0}^{t} D\left(t-t^{\prime}\right) \frac{\mathrm{d} n\left(t^{\prime}\right)}{\mathrm{d} t^{\prime}} \mathrm{d} t^{\prime}\right\}$,

wobei $\Sigma \beta_{i} \exp \left[-\lambda_{i}\left(t-t^{\prime}\right)\right]=D\left(t-t^{\prime}\right)$ gesetzt ist.

Um die wärmetechnische Seite des Problems, die bislang nicht in Betracht gezogen wurde, behandeln zu können, haben wir zunächst zu berücksichtigen, daß $k_{\text {eff }}$, der Vermehrungsfaktor des endlichen Reaktors, bei Temperaturerhöhung abnimmt. Für das Folgende kann $k_{\text {eff }}$ in der Form dargestellt werden:

$$
k_{\text {eff }}=k_{\text {eff }}\left(T_{\mathrm{A}}\right)-\Gamma T
$$

$k_{\text {eff }}\left(T_{\mathrm{A}}\right)=$ Vermehrungsfaktor bei Außentemperatur $T_{\mathrm{A}}\left(\right.$ ca. $\left.18^{\circ} \mathrm{C}\right)$,

$\Gamma=$ Temperaturkoeffizient des Reaktors,

$T=$ Temperatur oberhalb der Au Bentemperatur.

Für die Veränderung der inneren Wärmeenergie des Reaktors gilt ${ }^{2}$ :

$$
E \frac{\mathrm{d} T}{\mathrm{~d} t}=Q-A T
$$

$E=$ Wärmekapazität des Reaktors bzw. desjenigen Reaktorteiles, der bei Ausfall der Kühlung die Leistung aufnimmt,

$Q=$ im Reaktor erzeugte Leistung,

$A=$ Wärmeübergangszahl für Wärmeübergang an das Kühlmittel.

${ }^{2}$ H. Gaus u. R. Schulten, Z. Naturforschg. 9a, 964 [1954]. 
Diese Gleichung sagt aus, daß die Änderung der inneren Energie des Reaktors durch seine Leistung vergrößert und durch die Leistungsentnahme mittels der Kühlanlage [s. zweiter Summand der Gl. (7)] verkleinert wird.

Die strenge Proportionalität von $n$ und $Q$ gestattet, die Gln. (7) und (5) miteinander zu verbinden. Führt man als Abkürzung die Reaktivität

$$
k_{\text {eff }}\left(T_{\mathbf{A}}\right)--1=\delta k
$$

ein, so wird aus den Gln. (5) und (7):

$t_{0} \frac{\mathrm{d} Q}{\mathrm{~d} t}=(\delta k-\Gamma T) Q-k_{\mathrm{eff}} \int_{0}^{t} D\left(t-t^{\prime}\right) \frac{\mathrm{d} Q\left(t^{\prime}\right)}{\mathrm{d} t^{\prime}} \mathrm{d} t^{\prime},(9 \mathrm{a})$ $E \frac{\mathrm{d} T}{\mathrm{~d} t}=Q-A T$.

\section{Die Anfangsbedingungen}

Bis zum Zeitpunkt $t=0$ möge der Reaktor mit der Reaktivität $\delta k_{0}$ stationär betrieben werden. Dann folgen aus:

$$
\mathrm{d} Q / \mathrm{d} t=0 \text { und } \mathrm{d} T / \mathrm{d} t=0
$$

die Werte

$$
T_{0}=\frac{\delta k_{0}}{\Gamma} \text { und } Q_{0}=A T_{0}=A \frac{\delta k_{0}}{\Gamma} .
$$

Temperatur und Leistung des Reaktors sind also im stationären Fall durch die Überschußreaktivität, die Temperaturkonstante und die Wärmeübergangszahl des Reaktors eindeutig bestimmt.

Zum Zeitpunkt $t=0$ möge nun plötzlich die Kühlung aussetzen (d. h. $A=0$ ) und dabei die Reaktivität eine Veränderung erleiden (d. h. $k_{\text {eff }_{1}}=1+\delta k_{1} ; \delta k_{1} \gtrless \delta k_{0}$ ). Unter diesen Bedingungen heißen die Reaktorgleichungen

$t_{0} \frac{\mathrm{d} Q}{\mathrm{~d} t}=\left(\delta k_{1}-\Gamma T\right) Q-k_{\mathrm{eff}_{1}} \int_{0}^{t} D\left(t-t^{\prime}\right) \frac{\mathrm{d} Q\left(t^{\prime}\right)}{\mathrm{d} t^{\prime}} \mathrm{d} t^{\prime}$ $E \frac{\mathrm{d} T}{\mathrm{~d} t}=Q$

mit den Anfangsbedingungen

$$
T(0)=T_{0}=\frac{\delta k_{0}}{\Gamma} \text { und } Q(0)=Q_{0}=A \frac{\delta k_{0}}{\Gamma} .
$$

Dabei ist in (11a) im letzten Summanden $\Gamma T$ (Größenordnung $10^{-3}$ ) gegen $k_{\text {eff }}$ vernachlässigt worden.

Aus Gl. (11 b) sieht man unmittelbar, daß $T$ nur ansteigen kann. Dagegen kann nach Gl. (11a)
$Q$ im Zeitpunkt $t=0$ sowohl größer als auch kleiner werden, je nachdem ob

$$
\delta k_{1}-\Gamma T_{0}=\left(\delta k_{1}-\delta k_{0}\right)>\text { oder }<0 \text { ist. }
$$

Nach den Ausführungen in der Einleitung ist im ersten Falle der Reaktor inhärent instabil, im zweiten Falle inhärent stabil. Da nun $T$ immer größere Werte annimmt, wird von einem bestimmten Zeitpunkt an der erste Summand in Gl. (11 a) negativ werden, wenn er nicht, wie im stabilen Fall, von vornherein negativ ist. Infolgedessen wird auch $Q$ immer mehr abnehmen und schließlich gegen Null gehen. Für die zeitliche Änderung von $Q$ und $T$ erhält man also qualitativ die in Abb. 1 und 2 dargestellten Kurven.

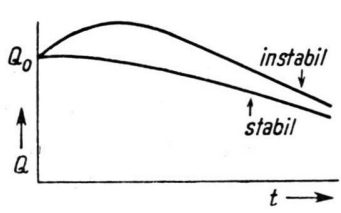

Abb. 1.

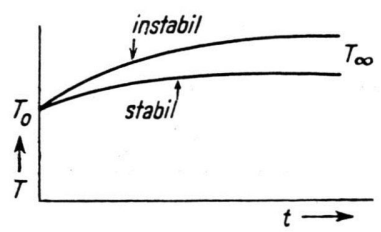

Abb. 2.
Abb. 1. Leistung in Abhängigkeit von der Zeit.

Abb. 2. Temperatur in Abhängigkeit von der Zeit.

\section{Bestimmung des Temperaturanstieges}

Unsere Aufgabe besteht nun darin, die Differenz $T_{\infty}-T_{0}$ aus den Reaktorgleichungen zu bestimmen. Dazu werde $Q$ aus Gl. (11 b) in Gl. (11 a) eingesetzt und von 0 bis $t$ integriert:

$$
\begin{aligned}
t_{0} Q & =\delta k_{1} E T-\frac{1}{2} \Gamma E T^{2} \\
& -k_{\mathrm{eff}_{1}} \int_{0}^{t} \mathrm{~d} t^{\prime} \int_{0}^{t^{\prime}} \mathrm{d} t^{\prime \prime} D\left(t^{\prime}-t^{\prime \prime}\right) \frac{\mathrm{d} Q\left(t^{\prime}\right)}{\mathrm{d} t^{\prime}}+C .
\end{aligned}
$$

$C$ ist eine Integrationskonstante, deren Wert aus Gl. (12) entnommen werden kann, wenn $t=0$ gesetzt wird

$$
C=t_{0} Q_{0}+\frac{1}{2} \Gamma E T_{0}^{2}-\delta k_{1} T_{0} E .
$$

Da $Q(\infty)=0$ ist, wird aus Gl. (12) für $t \rightarrow \infty$

$$
\begin{aligned}
0=\delta k_{1} E & T_{\infty}-\frac{1}{2} \Gamma E T_{\infty}{ }^{2} \\
& -k_{\mathrm{eff}_{1}} \int_{0}^{\infty} \mathrm{d} t^{\prime} \int_{0}^{t^{\prime}} \mathrm{d} t^{\prime \prime} D\left(t^{\prime}-t^{\prime \prime}\right) \frac{\mathrm{d} Q\left(t^{\prime}\right)}{\mathrm{d} t^{\prime}} \\
& +t_{0} Q_{0}+\frac{1}{2} \Gamma E T_{0}{ }^{2}-\delta k_{1} E T_{0} .
\end{aligned}
$$


Außerdem ist

$$
\begin{aligned}
& \int_{0}^{\infty} \mathrm{d} t^{\prime} \int_{0}^{t^{\prime}} \mathrm{d} t^{\prime \prime} D\left(t^{\prime}-t^{\prime \prime}\right) \frac{\mathrm{d} Q\left(t^{\prime}\right)}{\mathrm{d} t^{\prime}} \\
& \quad=\int_{0}^{\infty} \mathrm{d} t^{\prime \prime} \int_{t^{\prime \prime}}^{\infty} \mathrm{d} t^{\prime} D\left(t^{\prime}-t^{\prime \prime}\right) \frac{\mathrm{d} Q\left(t^{\prime}\right)}{\mathrm{d} t^{\prime}}=\sum_{i} \frac{\beta_{i}}{\lambda_{i}} Q_{0} .
\end{aligned}
$$

So folgt aus Gl. (14) für die Erhöhung der Temperatur

$$
\begin{aligned}
T_{\infty}-T_{0}=(1 / \Gamma) & \left\{\left(\delta k_{1}-\delta k_{0}\right)\right. \\
& \left.+\sqrt{\left(\frac{2 Q_{0} \Gamma}{E}\right) \bar{t}+\left(\delta k_{1}-\delta k_{0}\right)^{2}}\right\} .
\end{aligned}
$$

Hierbei wurde folgende Abkürzung eingeführt:

$$
\bar{t}=t_{0}+k_{\text {eff }_{1}} \sum_{i} \beta_{i} / \lambda_{i} .
$$

Das in Gl. (16) ausgedrückte Ergebnis zeigt, daß der betrachtete Prozeß - das plötzliche Abschalten der Kühlanlage mit gleichzeitiger Veränderung der Reaktivität - auf jeden Fall zu einer Temperaturerhöhung führt (d. h. $T_{\infty}-T_{0}>0$ ). Für negative $\left(\delta k_{1}-\delta k_{0}\right)$ bekommt man geringere Temperaturerhöhungen.

Ist $\delta k_{1}=\delta k_{0}$, so findet ein Temperaturanstieg statt, der von dem Verhältnis zwischen der stationären Leistung und der Wärmekapazität abhängt. Die Größe $\bar{t}$ hat für ${ }^{3}{ }^{235} \mathrm{U} ;{ }^{233} \mathrm{U} ;{ }^{233} \mathrm{Pu}$ resp. ungefähr die Werte 0,$1 ; 0,025 ; 0,033$ sec. Deshalb werden Reaktoren, die ${ }^{233} \mathrm{Pu}$ oder ${ }^{233} \mathrm{U}$ als Brennmaterial benutzen, kleinere Temperaturerhöhungen haben.

Besondere Verhältnisse liegen beim homogenen Leichtwasserreaktor (water boiler) vor, der ein in Wasser aufgelöstes Uransalz als Brennstoff enthält. Die Wärme wird in diesem Reaktor durch Umpumpen der gesamten Brennstofflösung entzogen. Gleichzeitig wird dabei ein großer Teil der latenten Neutronen aus dem Reaktor herausbefördert. Daher ist $\bar{t}$ für diesen Reaktor besonders klein.

In Abb. 3 ist der Zusammenhang zwischen $\left(\delta k_{1}-\delta k_{0}\right)$ und $\left(T_{\infty}-T_{0}\right)$ für Graphitreaktoren mit natürlichem Uran dargestellt. Dabei ist für $E$ lediglich die Wärmekapazität des Urans eingesetzt, d. h. es ist angenommen, daß die Uranstäbe praktisch wärmeisoliert sind. Dann kann der Ausdruck

\footnotetext{
${ }^{3}$ R. Stephenson, Introduction to Nuclear Engineering, Mc Craw-Hill Book Company. Inc., London 1954 .
}

$Q_{0} / E$ auf folgende Art durch den stationären Fluß $\Phi_{0}$ dargestellt werden

$\frac{Q_{0}}{E}=\frac{\text { erzeugte Leistung im Uran }}{\text { Wärmekapazität des Urans }}=\frac{\Sigma_{\mathrm{f}} \cdot \Phi_{0} \cdot 180 \mathrm{MeV}}{c \cdot \varrho}$,

wo $\varrho$ die Dichte und $c$ die spez. Wärme des Urans pro Gramm bedeuten. Als stationärer Fluß wurde $10^{11}$ und $10^{12} \mathrm{~cm}^{-2} \mathrm{sec}^{-1}$ zugrunde gelegt.

\section{Abschätzung des zeitlichen Ablaufes}

Nun möge noch die Dauer des oben beschriebenen Vorganges abgeschätzt werden. Grob gesprochen ist sie durch das Verhalten der langlebigsten verspäteten Neutronen bedingt. Diese stellen nach Gl. (2) im stationären Fall wegen ihrer langen Lebensdauer (d. h. wegen ihres kleinen $\lambda_{i}$ ) einen groBen Anteil an der Gesamtheit der zur Zeit $t=0$ vorhandenen latenten Neutronen. Die Leistung des Reaktors wird deshalb langsamer abklingen als die Konzentration dieser latenten Neutronen, deren mittlere Lebensdauer $83 \mathrm{sec}$ beträgt.

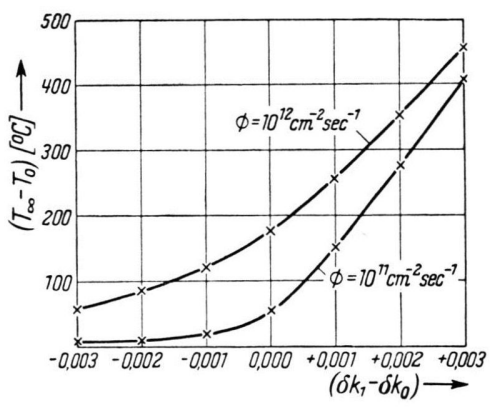

Abb. 3. Temperaturerhöhung für Graphitreaktoren in Abhängigkeit von der Reaktivität.

\section{Temperaturerhöhung durch verspätete $\gamma$ - und $\beta$-Strahlung}

Wie früher bemerkt, wird ein Teil der bei der Spaltung freiwerdenden Energie in Form einer verzögerten $\gamma$ - bzw. $\beta$-Strahlung produziert. Dieser Anteil, der pro Spaltung $10 \mathrm{MeV}$ (5\% der Gesamtenergie) beträgt, kann wegen seiner Verzögerung auch noch nach dem Ausfall der Kühlung und dem darauf folgenden Abklingen der Leistung zu einem beträchtlichen Anwachsen der Temperatur führen. Auch nach dem Zeitpunkt des Ausfalls der Kühlung werden im Reaktor noch Spaltprodukte produziert, da die Kettenreaktion, wie im vorigen Abschnitt gezeigt wurde, nicht sofort abreißt. Die Vermehrung der Leistung durch die $\gamma$ - 
und $\beta$-Strahlen dieser (nach dem Ausfall der Kühlung entstehenden) Spaltprodukte darf aber gegenüber der im vorigen Abschnitt behandelten Leistung wegen seines geringen Betrages (etwa 5\%) vernachlässigt werden.

Uns interessiert also nur die Leistung der verzögerten $\gamma$ - und $\beta$-Strahlen, die durch die vor dem Ausfall der Kühlung entstandenen Spaltprodukte produziert wird. Für die Zeit des Abklingens der Leistung im Reaktor nehmen wir hierfür als vorsichtige Abschätzung an, daß die Leistung der verzögerten Strahlung zeitlich konstant ist und den gleichen Betrag wie im vorausgehenden stationären Betrieb hat (10 MeV pro Spaltung). Die zusätzliche Temperaturerhöhung ist dann für diesen Zeitraum gegeben durch:

$$
E \frac{\mathrm{d} T_{\beta, \gamma}}{\mathrm{d} t}=\Sigma_{\mathrm{f}} V \Phi_{0} \cdot(10 \mathrm{MeV}),
$$

wobei $\Sigma_{\mathrm{f}}$ der makroskopische Absorptionsquerschnitt für Spaltung, $\Phi_{0}$ der stationäre Fluß und $V$ das Uranvolumen ist. Nimmt man (als ungünstigsten Fall) an, daß die entstehende Wärme nur vom Uran aufgenommen wird, so ergibt sich hieraus für natürliches Uran

$$
T_{\beta, \gamma}=t \cdot \Phi_{0} \cdot 1,3 \cdot 10^{-13}{ }^{\circ} \mathrm{C},
$$

wenn $t$ in sec und $\Phi_{0}$ in $\mathrm{cm}^{-2} \mathrm{sec}^{-1}$ gemessen wird.
Für reines ${ }^{235} \mathrm{U}$ ergibt sich

$$
T_{\beta, \gamma}=t \cdot \Phi_{0} \cdot 190 \cdot 10^{-13}{ }^{\circ} \mathrm{C} .
$$

Während bei der früher berechneten Maximaltemperatur die Wurzel aus der Leistung bzw. aus dem Fluß eingeht, ist dieser Temperaturanstieg dem Fluß direkt proportional. Hiernach würde z. B. nach 3 Minuten zu den in Abb. 3 angegebenen Temperaturen $2,2^{\circ} \mathrm{C}\left(\Phi_{0}=10^{11} \mathrm{~cm}^{-2} \mathrm{sec}^{-1}\right)$ bzw. $22{ }^{\circ} \mathrm{C}\left(\Phi_{0}=10^{12} \mathrm{~cm}^{-2} \mathrm{sec}^{-1}\right) \mathrm{zu}$ addieren sein. Bei Reaktoren mit größeren Flüssen, besonders bei angereichertem ${ }^{235} \mathrm{U}$, kann der Temperaturanstieg durch verzögerte Strahlung den früher berechneten wesentlich übertreffen.

Way und Wigner ${ }^{4}$ haben für die verzögerte Leistung einer Spaltung eine empirische Formel abgeleitet, die einen Gültigkeitsbereich von etwa einer Minute bis 100 Tagen hat. Wendet man diese Formel an, so ergibt sich für die Gl. (20) und (21) statt des linearen Anstieges in $t$ ein schwächerer Anstieg mit $(t)^{0,8}$. Wir können daher die obigen Gleichungen als eine obere Grenze betrachten.

Herrn Prof. K. Wirtz möchten wir für die Anregung zu der vorliegenden Untersuchung vielmals danken.

${ }^{4}$ K. Way u. E. P. Wigner, Phys. Rev. 73, 1318 [1948].

\title{
Wachstumskurven bei großen optischen Dicken
}

\section{Von Lennart Huldt}

Aus dem Institut für Physik der Universität Stockholm

(Z. Naturforschg. 9a, 1043-1047 [1954]; eingegangen am 26. September 1954)

\begin{abstract}
Wachstumskurven, welche die Beziehung zwischen Spektrallinienintensität - in Emission oder Absorption - und optischer Dicke darstellen, sind bis jetzt unter Annahme einer Überlagerung von Doppler-Form und Resonanzform der Spektrallinie berechnet worden. In größerer Entfernung von der Linienmitte weicht aber die Linienkontur von der einfachen Resonanzverteilung ab. Dies führt bei großen optischen Dicken zu einer von den früheren Resultaten etwas abweichenden Form der Wachstumskurven. Eine Berechnung dieser unter Zugrundelegung von Lindholms Druckverbreiterungsformeln, die sich experimentell gut bestätigt haben, wird hier für den Bereich durchgeführt, in welchem das Verhalten der äußeren Linienflügel entscheidend ist.
\end{abstract}

$\mathrm{D}$ ie Äquivalentbreite $W$ einer Spektrallinie der Wellenzahl $\sigma_{0}$ kann geschrieben werden:

$$
W=E / I_{0}\left(\sigma_{0}\right)
$$

wo im Falle der Absorption $E$ die gesamte absorbierte Energie und $I_{0}\left(\sigma_{0}\right)$ die Intensität der einfallenden Strahlung (über den Bereich der Linie als konstant angenommen) bedeuten. Im Falle der
Emission bedeutet $E$ die Energie der emittierten Linie und $I_{0}\left(\sigma_{0}\right)$ die Intensität der Planckschen Strahlung bei der Wellenzahl $\sigma_{0}$ und der gegebenen Lichtquellentemperatur, welch letztere allerdings nur formal zu sein braucht.

In beiden Fällen ist die Äquivalentbreite durch den Ausdruck gegeben:

$$
W=\int\left(1-e^{-\kappa(\sigma) \tau}\right) \mathrm{d} \sigma,
$$

